\title{
Consideration of the Influence of Independent Factors on Inspection Results and Reliability Updating of Marine Structures
}

\author{
by Alexandre Kawano*, Oscar Brito Augusto*
}

\begin{abstract}
A method for reliability updating of a group of similar structures is presented. The sources for variation among inspection results of structural details are divided into the effect of the particular structure a detail is imbedded in, the effect of the detail type, and the effect of a modeling error term. By using the proposed method, a deeper understanding of the causes of the differences among inspection results is possible, and as a consequence, a better inspection planning can be made.

Furthermore, since data of several structures and details are investigated at the same time, faster convergence rates to the true values are obtained than if just one individual structure is considered, making it possible for an owner or operator to keep the reliability of his fleet above a specified minimum level more cost effectively.
\end{abstract}

List of symbols

$N:$ Number of cycles till crack initiation.

$\Delta \sigma:$ Constant stress amplitude of the stress loading.

$Q$ and $p$ : Material characteristics concerning fatigue crack initiation.

$\Delta$ and $\Delta_{\text {crit }}$ : Accumulated fatigue damage.

$\sigma_{x}:$ Standard deviation of the stress process.

$E\left[N_{+}(0)\right]$ : Number of zero crossings from below per year of the stress process.

$T_{c}$ : Time to crack initiation.

$f_{T_{c}}(\quad)$ : Probability density function of the time to crack initiation.

$f(A \mid B)$ : Probability density function of the event A conditional to the occurrence of the event $\mathrm{B}$.

$S$ : Loading parameter.

$\alpha$ and $\beta$ : Shape and scale parameter of the Weibull variate used to randomize the crack initiation time.

$a:$ Crack size.

$a(\quad)$ : Crack size function.

$n:$ Number of stress cycles.

$\Delta K:$ Stress intensity factor range.

$C, K_{0}:$ Material properties associated to the Paris law. $a_{0}:$ Initial crack size.

$D(\quad)$ : Probability of crack detection function.

$d$ : Crack detection parameter.

$T_{h}$ : Last inspection time.

$T_{k}$ : First inspection time. Normally, $T_{k}=0$.

* Escola Politecnica, University of Sao Paulo, akawano@usp.br

Received 9th Jan. 1998

Read at the Spring meeting 14, 15th May 1998 $t_{c}(\quad)$ : Deterministic absolute crack initiation time.

$P_{1}\left(a, T_{h}, T_{k}\right)$ : Probability of finding a crack of size a at an inspection at time $T_{h}$ in a detail that started service at $T_{k}$, irrespective of its previous maintenance history.

$m_{c}$ and $c v_{c}$ : Median and coefficient of variation of the material parameter $C$.

$m_{s}, c v_{s}:$ Median and coefficient of variation of the loading parameter $S$.

$P_{2}\left(T_{h}, T_{k}\right)$ : Probability of not finding a crack in a detail at time $T_{h}$ in a detail that started service at $T_{k}$, irrespective of its previous maintenance history.

$P\left(A_{i j}, T_{h}, T_{k}\right)$ : Probability of the occurrence of the event $A_{i j}$ at time $T_{h}$. The time origin is at $T_{k}$.

$n_{i j}:$ Number of inspected details of the $j$-th type in the $i$-th structure.

$\beta_{i j}$ and $m_{s i j}: \beta$ and $m_{s}$ (see above) associated with the $j$-th detail type in the $i$-th structure.

$\bar{\beta}$ and $\overline{m_{s}}$ : Characteristic values of $\beta_{i j}$ and $m_{s i j}$.

$\beta_{s i}$ and $m_{s s i}$ : Effects (influences) of the $i$-th structure on $\beta_{i j}$ and $m_{s i j}$.

$\beta_{d j}$ and $m_{s d j}$ : Effects of the $j$-th detail type on $\beta_{i j}$ and $m_{S i j}$.

$\beta_{p_{i j}}$ and $m_{s p i j}$ : Effects of factors not explained by differences of structures or detail types on $\beta_{i j}$ and $m_{S i j}$.

$c v_{\beta S}$ and $c v_{S s}$ : Coefficients of variation of the effects of the structures on $\beta$ and $m_{s}$.

$c v_{\beta d}$ and $c v_{S d}$ : Coefficients of variation of the effects of the detail types on $\beta$ and $m_{s}$.

$c v_{\beta p}$ and $c v_{s p}$ : Coefficients of variation of the effects of factors not explained by differences among 
structures nor differences among detail types on $\beta$ and $m_{s}$.

$f^{(1)}(\quad)$ : A Bayesian prior probability density function. $f^{(0)}(\quad)$ : A Bayesian posterior density function.

Like $_{\text {I, II, II, IV }}\left(\alpha, \bar{\beta}, \overline{m_{s}} \mid A_{i j}\right)$ : Likelihood function of the parameters, $\alpha, \bar{\beta}$ and $\overline{m_{s}}$ (characteristic values) taking into account data of the regions I, II, III and IV.

$\beta_{d b}$ and $m_{s d b}:$ Effects of the $b$-th detail type on $\beta_{a b}$ and $m_{\text {sab }}$.

$\operatorname{Like}_{\mathrm{II}, \mathrm{IV}}\left(\beta_{d b}, m_{s d b} \mid A_{i j}\right)$ : Likelihood function of the parameters $\beta_{d b}$ and $m_{s d b}$ (effects of the $b$-th detail type) taking into account data of the regions II and IV.

$\beta_{s a}$ and $m_{s s a}$ : Effects of the $a$-th structure on $\beta_{a b}$ and sab.

$\beta_{p a b}$ and $m_{S p a b}$ : Effects of factors not explained by differences among structures nor detail types on $\beta_{a b}$ and $m_{s a b}$.

$\operatorname{Like}_{\text {Iv }}\left(\beta_{p a b}, m_{\text {spab }} \mid A_{a b}\right)$ : Likelihood function of the parameters $\beta_{p a b}$ and $m_{s p a b}$ (effects not covered by the model) taking into account data of the region IV.

$R_{1}(\quad)$ : Reliability of a detail that was repaired at the last inspection time.

$R_{2}(\quad)$ : Reliability of a detail that was not repaired at the last inspection time.

$R_{p q}(\quad)$ : Reliability of the set of detail type $q$ of the $p$ th structure.

\section{Introduction}

At the end of the 20th century, mankind is increasingly sensitive to disasters involving engineering structures that lead to death or any kind of environmental damage.

In the case of large steel structures under repetitive random loading such as ships and offshore installations, fatigue is often the governing limit state. Failure by fatigue in general has severe consequences since fatigue cracks can potentially grow unnoticed until it reaches critical sizes. By performing inspections at critical structural details, cracks may be detected, so that a minimum reliability level can, in principle, be maintained.

There are two ways by which inspections can be used to maintain or even improve the reliability levels. The first, more intuitive, as was suggested above, is that if a fatigue crack is detected, it is repaired and the original structural strength tends to be restored. The second, is that the inspection results themselves provide information for the statistical updating of uncertain parameters [Itagaki and Yamamoto, 1985].

However, as it is well known, the cost of inspections is a heavy burden to the owners or operators of such structures. For example, Vienneau[Vienneau, 1994] informs that for a VLCC, the cost of the Special survey no. 5 is estimated at U $\$ 5-7$ millions. Further, in general, the size of the structures involved makes the throughout inspection of all significant details impossible. For example,[Bea, 1991] reports that in a VLCC there may be almost one million square meters of steel, 400.000 meters of welds and 65,000 meters of stiffeners to search for critical damage. Due to the costs and time that the inspection task would take, even in special surveys, not all the structure is inspected, but parts of it that are judged to be representative of the whole behavior.

There is therefore a need for optimization of the inspection process, in terms of where to inspect, how much to inspect (extent) and when to inspect. Several researchers have already given several contributions to the field. Yang \& Trapp[Yang and Trapp, 1974] studied the effect of periodic inspections using classic probabilistic methods; Itagaki \& Yamamoto[Itagaki and Yamamoto, 1985] realizing that the inspection results themselves furnish data that can be used to update the knowledge about uncertain parameters, applied Bayesian analysis into the problem to select inspection intervals; Sorensen at alli[Sorensen at alli, 1991]proposed a Method to minimize overall service costs. Fujimoto[Fujimoto, 1990] proposed a method to obtain near-optimal solutions for the timing and the effort put in the inspection process.

As it was pointed out by Kai-tung Ma and others[Kai -tung Ma, 1997], there are real indications that correlation between inspection findings among different tanks of a same ship and among similar ships exists.

In this work, it is considered an updating scheme not for just one isolated structure, but for a group of similar ones from where it is expected some statistical correlation among structural behaviors and among inspection findings. A Bayesian version of the classical factorial experiment [Montgomery, 1976] approach with two factors, namely different individual structures and different structural detail types respectively is proposed.

By using information from the fleet as a whole, the reliability of the fleet can be maintained above a prespecified value with less expenditure of resources that if each ship were considered individually. Furthermore, by understanding the effects of the structure and the detail type on the fatigue life, better inspection plans can be devised.

\section{Mathematical Modeling}

Fatigue life is modeled as consisting of three distinct phases: crack initiation, propagation and failure.

\section{1 Crack initiation}

Crack initiation time is predicted with the use of $S$ $N$ curves, that relate a certain constant stress amplitude level to the corresponding number of cycles representing the number of cycles to crack initiation. To relate constant stress amplitude data to the real stochastic process stress loading $X(t)$, it is supposed that the Palmgrem-Miner rule applies, and an algorithm such as the rain flow counting can be used. 
A crack is said to initiate when it reaches a macro size $a_{0}$ from which phenomenological fracture mechanics is applicable, i.e., stress intensity factor amplitude becomes meaningful for describing the crack propagation phase.

Mathematically, the crack starting time (number of cycles) is supposed to be governed by the Wöhler formula :

$$
N(\Delta \sigma)^{p}=Q
$$

where $N$ is the number of cycles till crack initiation, $\Delta \sigma$ is the constant stress amplitude of the loading, and $Q$ and $\mathrm{p}$ are material characteristics. As it is well known, fatigue test data show great scatter. It could be modeled by making $p$ and $Q$ random variables, but instead, what is usually done[Pittaluga, Cazzulo \& Romeo, 1991] is to fix the value of $\mathrm{p}$ and model all randomness contained in these data by $Q$ only.

According to the $P-M$ rule, crack starts when the total damage $\Delta$ eaches the critical value $\Delta_{\text {crit. }}$ In the case of a stochastic stress process $X(t), \Delta$ is calculated as follows:

$$
\begin{aligned}
\Delta & =\sum_{j} \Delta_{j}=\sum_{j} \frac{n_{j}}{Q\left(\Delta \sigma_{j}\right)^{-p}} \\
& =\frac{1}{Q} \sum_{j} n_{j}\left(\Delta \sigma_{j}\right)^{p}=\frac{1}{Q} \sum_{j} n_{j}\left[\Delta X_{j}\right]^{p}
\end{aligned}
$$

where $n_{j}$ is the number of cycles corresponding to the constant stress amplitude $\Delta \sigma_{j}=\Delta X_{j}$. Making the assumption that the stress process $X(t)$ is a narrow band Gaussian process, and that the number of cycles is very high, the coefficient of variation (c. o. v.) of the summation factor is very small, and it is possible to approximate it by its mean value.

$$
\begin{aligned}
E\left[\sum_{j} n_{j}\left[\Delta X_{j}\right]^{p}\right]= & T \times E\left[N_{+}(0)\right](2 \sqrt{2})^{p}\left[\sigma_{x}\right]^{p} \\
& \Gamma\left(1+\frac{p}{2}\right)
\end{aligned}
$$

where in the formulas above, $T$ is the time in years, $\sigma_{x}$ is the standard deviation of the stress process $X(t)$, and $E\left[N_{+}(0)\right]$ is the number of zero crossings from below per unit time. A crack is said to initiate when $\Delta$ reaches a critical value $\Delta_{\text {crit. }}$ Substituting (3) into (2) and solving $\Delta=\Delta_{\text {crit }}$ when $T=T_{c}$, the critical time can be written as :

$$
T_{c}=\frac{Q \Delta_{\text {crit }}}{E\left[N_{+}(0)\right](2 \sqrt{2})^{p}\left[\sigma_{x}\right]^{p} \Gamma\left(1+\frac{p}{2}\right)}
$$

The fatigue life itself presents great variability. Besides, there are several uncertainties in the evaluation of $\Delta_{\text {crit }}, Q$, and in other factors. Also, there are several uncertainties concerning the stress process.

To model them, two random variables are introduced. The first one is a random factor $Z$ that follows a 2 parameter Weibull distribution to model the scatter and the uncertainties in the fatigue life modeling. The other is a loading parameter $S$ which is assumed to follow a Lognormal distribution with median $m_{s}$ and coefficient of variation $c v_{s}$.

$$
T_{c}=\frac{Q \Delta_{\text {crit }} \times Z}{E\left[N_{+}(0)\right](2 \sqrt{2})^{p}\left[\sigma_{x}\right]^{p} \Gamma\left(1+\frac{p}{2}\right) \times S}
$$

The conditional probability density function of $Z$, given the knowledge of the values of the shape parameter $\alpha$ and the scale parameter $\beta$, is given by

$$
f_{z}(z \mid \alpha, \beta)=\left(\frac{\alpha}{\beta}\right)\left(\frac{z}{\beta}\right)^{\alpha-1} \exp \left[-\left(\frac{z}{\beta}\right)^{\alpha}\right]
$$

The conditional probability density function for the critical time $T_{c}$ can be easily found by simple transformation of variables:

$$
\begin{aligned}
f_{T_{c}}(t \mid \alpha, \beta, S)= & J \times S \times\left(\frac{\alpha}{\beta}\right)\left(\frac{t}{\beta / J \times S}\right)^{\alpha-1} \\
& \cdot \exp \left[-\left(\frac{t}{\beta / J \times S}\right)^{\alpha}\right]
\end{aligned}
$$

where

$$
J=\frac{E\left[N_{+}(0)\right](2 \sqrt{2})^{p}\left[\sigma_{x}\right]^{p} \Gamma\left(1+\frac{p}{2}\right)}{Q}
$$

The corresponding distribution function is given by

$$
F_{T_{c}}(t \mid \alpha, \beta, S)=1-\exp \left[-\left(\frac{t}{\beta / J \times S}\right)^{\alpha}\right]
$$

Note that in the above model, no use has been made of an 'endurance limit' that is observed in constant amplitude fatigue tests of steel specimens. In fact, the 'endurance limit' seems to vanish, or at least it seems to be suppressed to a very low level under variable amplitude loading[Sarkani et alli., 1994].

\section{2 Crack propagation}

After initiation, a crack is assumed to propagate according to the Paris law :

$$
\frac{d a}{d n}=C\left(\frac{\Delta K}{K_{0}}\right)^{p}
$$

where $a$ is the crack size, $n$ is the number of cycles, $\Delta K$ is the stress intensity factor range, and $C, p$ and $K_{0}$ are material properties.

In probabilistic reliability analysis the above formula is randomized in order to reflect the fairly high scatter present in crack propagation data see e.g.[Sobczyk K. \& Spencer, 1992] [Itagaki, Ishizuka \& Yan, 1993]. In this work, it is supposed that $p$ is deterministic, and $C$ is a lognormal random variable in line with the model proposed by Yang[Yang et alli, 1993]. The stress intensity factor $\Delta K$ is given by :

$$
\Delta K=\Delta X Y(a) \sqrt{\pi a}
$$

where $Y(a)$ is a non-dimensional geometry factor, which depends both on the structural detail and the shape and length of the crack. To simplify analysis, $Y(a)$, in this work is supposed to be constant and equal to one, which corresponds to the infinite plate solution.

Supposing that the stress process $\Delta X=\Delta X(t)$ is narrow-band Gaussian and integrating (10), the crack length $a$ as a function of time $t$ is obtained:

$$
\begin{aligned}
a\left(t-t_{c}, C, S\right)= & {\left[a_{0} \frac{2-p}{2}+\left(t-t_{c}\right) C \gamma S \frac{2-p}{2}\right.} \\
& \left.\cdot\left(\frac{\sqrt{\pi}}{K_{0}}\right)^{p}\right]^{\frac{2}{2-p}}, p \neq 2
\end{aligned}
$$

where $t_{c}$ stands for crack initiation time and: 


$$
\gamma=E\left[N_{+}(0)\right](2 \sqrt{2})^{p}\left[\sigma_{x}\right]^{p} \Gamma\left(1+\frac{p}{2}\right)
$$

In the above formula, $a_{0}$ is the initial crack size.

\section{3 Crack detection}

The probability of detecting an existing crack of size a is given by

$$
D(a)=1-\exp \left[-d\left(a-a_{0}\right)\right]
$$

where $d$ is a crack detection parameter.

\section{4 Events at the inspection times and their respective probabilities}

The Bayesian method is used to update the knowl edge about uncertain probabilistic parameters present in the mathematical model. Specifically, in this work, by the knowledge of inspection results, the degrees of belief or probability density functions of the crack initiation parameter $\beta$, the shape parameter $\alpha$, and the loading parameter $S$ are updated via the Bayes method.

To apply the Bayesian method, it is necessary first to describe what events are expected to be observed in the inspections. A structural detail is defined so that it has only one fatigue critical location where a crack can initiate. Besides, in each structure $i$, only $n_{i j}$ details of type $j$ are inspected.

The following events form the sample space for inspection results concerning one detail :

a) A crack of size $a^{1}$ is found in the detail.

b) No cracks are found in it.

The probability density function of the size of the crack at time $T_{h}$ in a detail that started service at time $T_{k}$ in given by

$$
f\left(a \mid T_{h}, T_{k}, C, \alpha, \beta, S\right)=\left\{\begin{array}{l}
f_{T_{c}}\left(t_{c}(a, C, S) \mid \alpha, \beta\right) \frac{d t_{c}}{d a}, \text { if } \\
t_{c}(a, C)>T_{k} \\
0, \quad \text { otherwise }
\end{array}\right.
$$

where $t_{c}(a, C, S)$ is the absolute time of crack initiation, obtained from (12), and given by

$$
t_{c}(a, C, S)=T_{h}-\frac{\left(a^{\frac{2-p}{2}}-a_{0}^{\frac{2-p}{2}}\right)}{\gamma \times C \times S \times\left(\frac{\sqrt{\pi}}{K_{0}}\right)^{p} \times \frac{2-p}{2}}
$$

The probability of detecting a crack of size a at time $T_{h}$ in a detail that was put into service at time $T_{k}$, and no cracks are detected till $T_{h}$ is given by

$$
\begin{aligned}
& p_{1}\left(a, T_{h}, T_{k} \mid \alpha, \beta, C, S\right) d a=f\left(a \mid T_{h}, T_{k}, C, \alpha, \beta, S\right) \\
& \quad \times \prod_{l=k+1}^{h-1}\left[1-H\left(T_{l}-t_{c}(a, C, S)\right)\right. \\
& \left.\quad \times D\left(a\left(T_{l}-t_{c}(a, C, S), C, S\right)\right)\right] \times D(a) d a
\end{aligned}
$$

The probability of finding a crack of any size at time $T_{h}$ in a detail that was put into service at time $T_{k}$ and no cracks are detected from then on is given by

$$
\begin{aligned}
p_{1}^{*} & \left(T_{h}, T_{k} \mid \alpha, \beta, C, S\right)=\int_{T_{k}}^{T_{h}} f_{T_{\mathrm{c}}}\left(t-T_{k} \mid \alpha, \beta, S\right) \\
& \times \prod_{l=k+1}^{h-1}\left[1-H\left(T_{l}-t\right) D a\left(a\left(T_{l}-t, C, S\right)\right)\right] \\
& \times D\left(a\left(T_{h}-t, C, S\right)\right) d t
\end{aligned}
$$

\footnotetext{
${ }^{1}$ it in fact means "a crack of size between a and $a$ $+d a "$
}

The probability $P_{r}$ of finding a crack, not considering the fact of previous detection and maintenance can be calculated from the formula above and the following recursive scheme if the inspections are equispaced in time :

$$
\begin{aligned}
& P_{r}\left(T_{h}, T_{k} \mid \alpha, \beta, C, S\right) \\
& \quad=\left\{\begin{array}{l}
\sum_{i=k+1}^{h-1}\left[\begin{array}{l}
p_{1}^{*}\left(T_{i}, T_{k} \mid \alpha, \beta, C, S\right) \times \\
P_{r}\left(T_{h}, T_{i} \mid \alpha, \beta, C, S\right)
\end{array}\right]+ \\
p_{1}^{*}\left(T_{h}, T_{k} \mid \alpha, \beta, C, S\right), \text { if }(h-k) \geq 2 \\
p_{1}^{*}\left(T_{h}, T_{k} \mid \alpha, \beta, C, S\right), \text { if }(h-k)=1
\end{array}\right.
\end{aligned}
$$

The probability $P_{1}$ da of finding a crack of size $a$ at an inspection at time $T_{h}$ in a detail that started service at $T_{k}$, not considering the fact of previous detection and maintenance is given by:

$$
\begin{aligned}
& P_{1}\left(a, T_{h}, T_{k} \mid \alpha, \beta, C, S\right) d a \\
&= p_{1}\left(a, T_{h}, T_{k} \mid \alpha, \beta, C, S\right) d a \\
& \quad+ \sum_{i=k+1}^{h-1} P_{r}\left(T_{i}, T_{k} \mid \alpha, \beta, C, S\right) \\
& \quad \times p_{1}\left(a, T_{h}, T_{i} \mid \alpha, \beta, C, S\right) d a
\end{aligned}
$$

It is assumed that both $C$ and $S$ follow lognormal distributions with parameters :

$$
C \sim L N\left(m_{c}, c v_{c}\right) \quad S \sim L N\left(m_{s}, c v_{s}\right)
$$

where $m_{c}$ and $m_{S}$ stand for the median of their respective distributions, and $c v_{c}$ and $c v_{s}$ for their coefficient of variation.

The probability given by (20) in terms of $m_{c}$ and $c v_{c}$ becomes

$$
\begin{aligned}
P_{1}\left(a, T_{h}, T_{k} \mid \alpha, \beta, m_{S}\right) d a & \\
= & \int_{0}^{+\infty} \int_{0}^{+\infty}\left\{P_{1}\left(a, T_{h}, T_{k} \mid \alpha, \beta, C, S\right)\right. \\
& \left.f_{C}(C) f_{C}\left(S \mid m_{S}\right)\right\} d C d S d a
\end{aligned}
$$

The probability of not detecting any cracks at $T_{h}$ in a detail that started service at $T_{k}$, is simply given by

$$
\begin{aligned}
P_{2} & \left(T_{h}, T_{k} \mid \alpha, \beta, m_{S}\right) \\
= & \int_{0}^{+\infty} \int_{0}^{+\infty}\left\{1-P_{r}\left(T_{h}, T_{k} \mid \alpha, \beta, C, S\right)\right. \\
& \left.f_{C}(C) f_{S}\left(S \mid m_{S}\right)\right\} d C d S
\end{aligned}
$$

2. 5 Information gathering from similar structures

Consider a group of similar structures that contain similar structural detail types subject to similar, but different, stress loadings. Since each member of the group of structures has been built with different construction teams, and is located at different places and therefore are subjected to different load histories, it is natural to imagine some definite influence of a particular structure, detail type, and loading history on the fatigue life, but it is also natural to expect some correlation between inspection results.

The probability of the event

$$
A_{i j}=\left\{\begin{array}{l}
\left(n_{i j}\right)_{1} \text { elements out of } n_{i j} \text { are found to } \\
\text { have cracks of sizes } a_{1}, \cdots, a_{\left(n_{i j}\right)_{1}}, \\
\text { and }\left(n_{i j}\right)_{2}=n_{i j}-\left(n_{i j}\right)_{1} \text { are found } \\
\text { to be intact (no cracks) in the } j \text {-th } \\
\text { detail of the } i \text {-th strucure }
\end{array}\right\}
$$

can be given by the expression 


$$
\begin{aligned}
& P\left(A_{i j}, T_{h}, T_{k} \mid \alpha, \beta, m_{S}\right) d a_{1} \cdots d a_{\left(n_{i j)}\right)}=\frac{n_{i j} !}{\left(n_{i j}\right)_{2} !} \times \\
& \quad\left(P_{2}\left(T_{h}, T_{k} \mid \alpha, \beta, m_{S}\right)\right)^{\left(n_{i j)}\right)} \prod_{l=1}^{\left(n_{i j}\right)} P_{1}\left(a_{l}, T_{h}, T_{k} \mid \alpha, \beta,\right.
\end{aligned}
$$

$\left.m_{S}\right) d a_{\imath}$

To account the influence of each particular structure and detail type, the values of $\beta$ and $m_{s}$ are partitioned into components or effects.

$$
\begin{aligned}
\beta_{i j}= & \bar{\beta} \times \beta_{s i} \times \beta_{d j} \times \beta_{p_{i j}} \quad m_{s i j}=\bar{m}_{S} \times m_{s s i} \times m_{s d j} \\
& \times m_{s p i j}
\end{aligned}
$$

where $\bar{\beta}$ and $\bar{m}_{s}$ are the characteristic values, $\beta_{s i}$ and $m_{s s i}$ are the effects of the $i$-th structure, $\beta_{d j}$ and $m_{s d j}$ are the effects of the $j$-th type of detail, and $\beta_{p i j}$ and $m_{s p i j}$ are the effects of other factors not explained by the model.

The probability density functions are as follows.

$$
\begin{gathered}
\beta_{s i} \sim L N\left(1, c v_{\beta S}\right) \beta_{d j} \sim L N\left(1, c v_{\beta d}\right) \beta_{p_{i j}} \sim L N\left(1, c v_{\beta p}\right) \\
\beta_{i j} \sim L N\left(\bar{\beta}, \sqrt{\left.\left(c v_{\beta S}^{2}+1\right)\left(c v_{\beta d}^{2}+1\right)\left(c v_{\beta p}^{2}+1\right)-1\right)}\right.
\end{gathered}
$$

Similarly

$$
\begin{aligned}
& m_{S s i} \sim L N\left(1, c v_{s s}\right) m_{S d j} \sim L N\left(1, c v_{s d}\right) m_{S p i j} \\
& \quad \sim L N\left(1, c v_{s p}\right) \\
& \quad m_{s i j} \sim L N\left(\overline{m_{s}}, \sqrt{\left(c v_{s s}^{2}+1\right)\left(c v_{s d}^{2}+1\right)\left(c v_{S p}^{2}+1\right)-1}\right)
\end{aligned}
$$

The probability of the event $A_{i j}$ at an inspection at time $T_{h}$ conditional on the uncertain parameters $\beta_{i j}$ and $m_{S i j}$ can be written as $P\left(A_{i j}, T_{h}, T_{k} \mid \alpha, \beta_{i j}, m_{s i j}\right)$, obtained by substituting $\beta$ and $m_{s}$ for $\beta_{i j}$ and $m_{s i j}$ in equation (24). Now, the probability of the event $A_{i j}$ in terms of the characteristic values $\bar{\beta}$ and $\overline{m_{s}}$, can be given by the integral

$$
\begin{aligned}
& P\left(A_{i j}, T_{h}, T_{k} \mid \alpha, \bar{\beta}, \overline{m_{S}}\right) \\
& \quad=\int_{0}^{+\infty} \int_{0}^{+\infty}\left\{P\left(A_{i j}, T_{h}, T_{k} \mid \alpha, \beta_{i j}, m_{S i j}\right)\right. \\
& \left.f_{\beta_{i j} m_{S i j}}\left(\beta_{i j}, m_{S i j} \mid \bar{\beta}, \overline{m_{S}}\right)\right\} d \beta_{i j} d m_{S i j}
\end{aligned}
$$

where $f_{\beta_{i j} m_{s i}}\left(\beta_{i j}, m_{s i j} \mid \bar{\beta}, \overline{m_{S}}\right)$ is described by (26) and (27), supposing that $\beta_{i j}$ and $m_{s i j}$ are independent.

Suppose we wish to collect all information available to infer the probabilistic parameters $\alpha, \beta_{a b}$ and $m_{s a b}$ about a specific type of detail $b$ in a specific structure $a$. This can be done by using the Bayesian Method.

Consider the diagram shown in figure 1. It is divided into four areas I, II, III, and IV. Area I contains all combinations $(i, j)$ of structure $i$ and type of detail $j$ such that $i \neq a$ and $j \neq b$. Area II corresponds to all cells of detail type $b$, except the one on the structure $a$. Area III contains all details of the structure $a$, except the detail type $b$. Finally, the area IV corresponds to the detail type $b$ on the structure $a$.

At the first level, to get information about $\alpha, \bar{\beta}$ and $\bar{m}_{s}$, the following Bayesian updating relation is used.

$$
\begin{aligned}
& f_{\alpha, \bar{\beta}, \overline{m_{s}}}^{(1)}\left(\alpha, \bar{\beta}, \overline{m_{S}}\right)= \\
& \frac{\text { Like }_{\mathrm{I}, \mathrm{II}, \mathrm{III}, \mathrm{IV}}\left(\alpha, \bar{\beta}, \overline{m_{S}} \mid A_{i j}\right) \times f_{\alpha, \beta, m_{s}}^{(0)}\left(\alpha, \bar{\beta}, \overline{m_{S}}\right)}{} \\
& \left\{\int_{m_{S}} \int_{\beta} \int_{\alpha} \operatorname{Like}_{\mathrm{I}, \mathrm{II}, \mathrm{III}, \mathrm{IV}}\left(\alpha, \bar{\beta}, \overline{m_{S}} \mid A_{i, j}\right)\right. \\
& \left.\quad \times f_{\alpha, \beta, m_{S}}^{(0)}\left(\alpha, \bar{\beta}, \overline{m_{s}}\right) d \alpha d \bar{\beta} d \overline{m_{s}}\right\}
\end{aligned}
$$

where $f_{\alpha, \bar{\beta}, \overline{m_{s}}}^{(0)}\left(\alpha, \bar{\beta}, \overline{m_{S}}\right)$ is the prior degree of belief

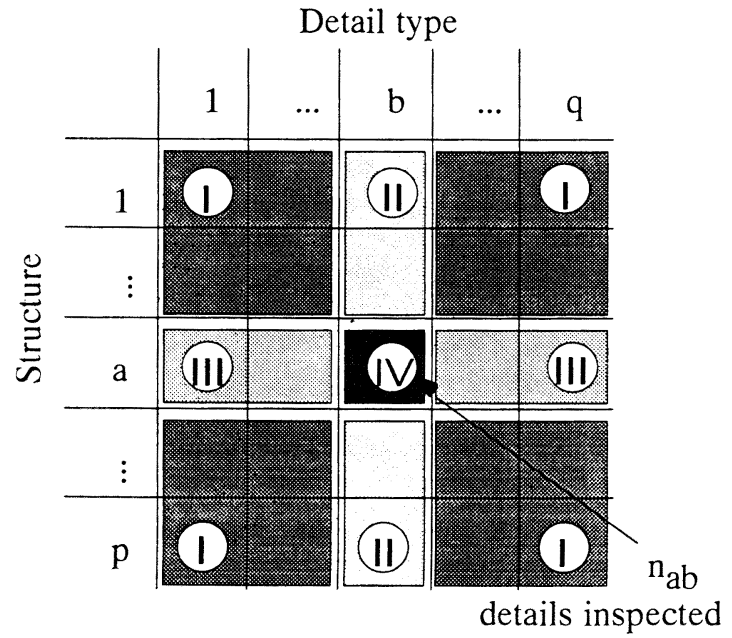

Fig. 1 Decomposition into four areas

distribution, $f_{\alpha, \bar{\beta}}^{(1)}, \overline{m_{S}}\left(\alpha, \bar{\beta}, \overline{m_{S}}\right)$, the posterior, and Like L $_{\mathrm{I}}$ II, III, IV $\left(\alpha, \bar{\beta}, \overline{m_{s}} \mid A_{i j}\right)$ is the likelihood of obtaining the data of regions I, II, III and IV.

The likelihood function $\operatorname{Like}_{\mathrm{I}, \mathrm{II}, \mathrm{III}, \mathrm{IV}}\left(\alpha, \bar{\beta}, \overline{m_{S}} \mid A_{i j}\right)$ is given by :

$$
\begin{aligned}
& \text { Like }_{\mathrm{I}, \mathrm{II}, \mathrm{III}, \mathrm{IV}}\left(\alpha, \bar{\beta}, \overline{m_{S}} \mid A_{i j}\right) \\
& \quad=\prod_{l=1}^{h} \prod_{i=1}^{p} \prod_{j=1}^{q} P\left(A_{i j}, T_{l}, T_{k} \mid \alpha, \bar{\beta}, \overline{m_{S}}\right)
\end{aligned}
$$

where $P\left(A_{i j}, T_{l}, T_{k} \mid \alpha, \bar{\beta}, \overline{m_{s}}\right)$ is given by (28), and normally $T_{k}=0$.

To get information about the parameters $\beta_{d b}$ and $m_{S d b}$, another likelihood function $\operatorname{Like}_{\mathrm{II}, \mathrm{IV}}\left(\beta_{d b}, m_{s d b} \mid A_{i b}\right)$ is used. The conditional probability of getting a result in a cell $(i, b)$ belonging to the regions II or IV is given by :

$$
\begin{aligned}
& P\left(A_{i b}, T_{h} \mid \alpha, \bar{\beta}, \overline{m_{s}}, \beta_{d b}, m_{s d b}\right)= \\
& \quad \int_{0}^{+\infty} \int_{0}^{+\infty} P\left(A_{i b}, T_{h} \mid \alpha, \bar{\beta} \times \beta_{d b} \times x, \overline{m_{S}} \times m_{S d b} \times y\right) \\
& \quad \times f_{x, y}(x, y) d x d y
\end{aligned}
$$

where $f_{x, y}(x, y)$ is the probability density function of $\left(\beta_{s i} \times \beta_{p i j}, m_{s s i} \times m_{S p i j}\right)$.

The likelihood function corresponding to the regions II and IV is given by :

$$
\begin{aligned}
& \left(\mathrm{Like}_{\mathrm{II}, \mathrm{IV}}\left(\beta_{d b}, m_{S d b} \mid A_{i b}\right)=\right. \\
& \int_{\alpha, \bar{\beta}, \overline{m_{S}}}\left\{\prod_{l=1}^{h} \prod_{i=1}^{p} P\left(A_{i b}, T_{l}, T_{k} \mid \alpha, \bar{\beta}, \overline{m_{S}}, \beta_{d b}, m_{S d b}\right)\right\} \\
& f_{\alpha, \bar{\beta}, \overline{m_{S}}}^{(1)}\left(\alpha, \bar{\beta}, \overline{m_{S}}\right) d \alpha d \bar{\beta} d \overline{m_{S}}
\end{aligned}
$$

The posterior density function of $\beta_{d b}$ is given by:

$$
\begin{aligned}
& f_{\beta d b}^{(1)}, m_{S d b}\left(\beta_{d b}, m_{S d b} \mid A_{i b}\right)= \\
& \frac{\operatorname{Like}_{\mathrm{II}, \mathrm{IV}}\left(\beta_{d b}, m_{S d b}\right) f_{\beta d, m_{S d b}}^{(0)}\left(\beta_{d b}, m_{S d b}\right)}{\int_{\beta d_{b}, m_{S d b}} \operatorname{Like}_{\mathrm{II}, \mathrm{IV}}\left(\beta_{d b}, m_{S d b}\right) f_{\beta d, m_{S d b}}^{(0)}\left(\beta_{d b}, m_{S d b}\right)}
\end{aligned}
$$

where $f_{\beta d, m_{S d b}}^{(0)}\left(\beta_{d b}, m_{s d b}\right)$ is the prior distribution of the parameters $\beta_{d b}$ and $m_{s d b}$. A consistent choice must be based on the distributions of (26) and (27). 
The posterior distribution $f_{\beta_{s a}, m_{s s a}}^{(1)}\left(\beta_{s a}, m_{s s a}\right)$ after observing the inspection results of the structure a (regions III and IV) can be obtained in a manner completely analogous as was done to obtain $f_{\beta_{d}, m_{S d b}}^{(1)}$ $\left(\beta_{d b}, m_{s d b}\right)$.

Finally, the posterior probability function for the residual effects term can be evaluated by observing the inspection results in the region IV.

$$
\begin{aligned}
& f_{\beta_{p a b}, m_{\text {spab }}}^{(1)}\left(\beta_{p a b}, m_{\text {Spab }}\right)= \\
& \operatorname{Like}_{\mathrm{LIV}}\left(\beta_{p a b}, m_{\text {Spab }} \mid A_{a b}\right) \times f^{(0)}\left(\beta_{p a b}, m_{\text {spab }}\right) \\
& \left\{\int_{\beta_{p a b}, m_{\text {Spab }}} \operatorname{Like}_{\mathrm{IV}}\left(\beta_{p a b}, m_{\text {Spab }} \mid A_{a b}\right)\right. \\
& \left.\quad \times f^{(0)}\left(\beta_{p a b}, m_{\text {Spab }}\right) d \beta_{\text {pab }} d m_{\text {Spab }}\right\}
\end{aligned}
$$

To be consistent, the prior distribution $f^{(0)}\left(\beta_{p a b}\right.$, $\left.m_{\text {Spab }}\right)$ must be given by (26) and (27). The numerator of (34) can be given by

$$
\begin{aligned}
& \operatorname{Like}_{\text {Iv }}\left(\beta_{p a b}, m_{s p a b} \mid A_{a b}\right)= \\
& \int\left\{\prod _ { l = 1 } ^ { h } P \left(A_{a b}, T_{l} \mid \alpha, \bar{\beta} \times \beta_{s a} \times \beta_{d b} \times \beta_{p a b}, \overline{m_{S}}\right.\right. \\
& \left.\quad \times m_{s s a} \times m_{S d b} \times m_{s p a b}\right) \times \\
& f_{\alpha, \beta, \sigma z}^{(1)}\left(\alpha, \bar{\beta}, \overline{m_{s}}\right) \times f_{\beta_{s a}, m_{s s a}}^{(1)}\left(\beta_{s a}, m_{s s a}\right) \\
& \left.\quad \times f_{\beta_{d b}, m_{s d b}}^{(1)}\left(\beta_{d b}, m_{s d b}\right)\right\} \\
& \quad \times d \alpha d \bar{\beta} d \overline{m_{s}} d \beta_{s a} d m_{s s a} d \beta_{d b} d m_{s d b}
\end{aligned}
$$

With more precise information about the uncertain parameters than in the beginning of the process, the planning of future inspections can be done more effectively, and the reliability kept at the required level with less expenditure of resources.

\section{6 Reliability Evaluation}

Reliability is defined here simply as the probability of the existence of a crack greater than a certain critical value acrit $a_{\text {crit. }}$. For the evaluation of that probability, it must be considered two types of details. Those in which a crack was found at the last inspection, and those in which no crack has been found. Assuming that after a repair, the detail condition is as good as new, the reliability of a detail that was repaired in the last inspection at $T_{h}$ is given by

$$
\begin{gathered}
R_{1}\left(t, T_{h} \mid \alpha, \beta, m_{s}\right)=\int_{0}^{+\infty} \int_{0}^{+\infty}\left(1-F_{T_{c}}\left(t-T_{h} \mid \alpha, \beta, S\right)\right) \\
+\int_{a_{0}}^{a_{\text {crit }}} f_{a}\left(a \mid t, T_{h}, C, \alpha, \beta, S\right) f_{C}(C) f_{S}\left(S \mid m_{s}\right) d a d C \\
\quad d S
\end{gathered}
$$

where $f_{a}\left(a \mid t, T_{h}, C, \alpha, \beta, S\right)$ is given by (15).

Now, the reliability of a detail that has not been repaired in the last inspection at $T_{h}$ is given by

$$
\begin{aligned}
& R_{2}\left(t, T_{h} \mid \alpha, \beta, m_{s}\right)=1 \\
& \quad P\left[\left(a>a_{\text {crit }} \text { at } t>T_{h}\right) \cap\right. \\
& -\frac{\left.\left(\text { no crack has been found at } T_{h}\right)\right]}{P\left[\text { no crack has been found at } T_{h}\right]} \\
& P\left[\left(a>a_{\text {crit at } \left.t>T_{h}\right) \cap(\text { no crack has been found at }}\right.\right. \\
& \left.\left.\quad T_{h}\right)\right]= \\
& \left\{\begin{array}{l}
p_{3}\left(t, T_{h}, T_{k} \mid \alpha, \beta, C, S\right) \\
+\sum_{i=k+1}^{n-1}\left[\begin{array}{l}
\left.P_{r}\left(T_{i}, T_{k} \mid \alpha, \beta, C, S\right) \times\right] \\
p_{3}\left(t, T_{h}, T_{i} \mid \alpha, \beta, C, S\right)
\end{array}\right], \text { if }(h-k) \geq 2 \\
p_{3}\left(t, T_{h}, T_{k} \mid \alpha, \beta, C, S\right), \text { if }(h-k)=1
\end{array}\right.
\end{aligned}
$$

$$
\begin{gathered}
p_{3}\left(t, T_{h}, T_{k} \mid \alpha, \beta, C, S\right)=\int_{T_{k}}^{t_{\mathrm{crit}}} f_{T_{c}}\left(t-T_{k} \mid \alpha, \beta, S\right) \\
\quad \times \prod_{l=k+1}^{h}\left[1-H\left(T_{l}-t\right) D\left(a\left(T_{l}-t, C, S\right)\right)\right] d t
\end{gathered}
$$

where $t_{\text {crit }}$ is the maximum absolute time corresponding to a crack of size $a_{\text {crit }}$, that is, any crack that initiates after $t_{\text {crit }}$ has size less than $a_{\text {crit. }}$. The function $p_{3}$ gives the probability of the existence of a crack greater than $a_{\text {crit }}$ and this crack is not detected before at previous inspections (including the one at $T_{h}$ ).

The denominator is simply given by (23).

\begin{tabular}{|c|c|c|c|c|}
\hline \multicolumn{2}{|c|}{$\begin{array}{l}\text { Physical } \\
\text { Parameter }\end{array}$} & Symbol & Value & Unit \\
\hline \multicolumn{2}{|c|}{$\begin{array}{l}\text { Mean value of } \\
\text { the crack } \\
\text { propagation } \\
\text { rate C }\end{array}$} & $\mu_{\mathrm{C}}$ & $1.0 \times 10^{-7}$ & $\mathrm{~m} /$ cycle \\
\hline \multicolumn{2}{|c|}{$\begin{array}{l}\mathrm{K}_{0} \text { parameter in } \\
\text { the crack } \\
\text { propagation } \\
\text { formula }\end{array}$} & $\mathrm{K}_{0}$ & $30.0 \times 10^{-6}$ & $\mathrm{~Pa} \sqrt{\mathrm{m}}$ \\
\hline \multirow{2}{*}{ 兽 } & Min, detect. & $a_{0}$ & 0.01 & $\mathrm{~m}$ \\
\hline & Critical & $a_{\text {crit }}$ & 0.3 & $\mathrm{~m}$ \\
\hline \multicolumn{2}{|c|}{$\begin{array}{l}\text { Detection } \\
\text { parameter d }\end{array}$} & d & 10.0 & $\mathrm{~m}^{-1}$ \\
\hline \multicolumn{2}{|c|}{$\begin{array}{l}\text { Paris law m } \\
\text { exponent }\end{array}$} & $\mathrm{m}$ & 3.0 & \\
\hline \multicolumn{2}{|c|}{$\begin{array}{l}\text { Mean Number of } \\
\text { cycles per year }\end{array}$} & $\mathrm{E}\left[\mathrm{N}_{+}(0)\right]$ & $5.0 \times 10^{6}$ & cycles/year \\
\hline \multicolumn{2}{|c|}{$\begin{array}{l}\text { Standard } \\
\text { Deviation of the } \\
\text { stress process }\end{array}$} & $\sigma_{\mathrm{x}}$ & $15 \times 10^{6}$ & $\mathrm{~Pa}$ \\
\hline \multicolumn{2}{|c|}{$\begin{array}{l}\text { Wöhler formula } \\
\text { eq. (1) }\end{array}$} & \multicolumn{2}{|c|}{$\begin{array}{c}N(\Delta \sigma)^{3}=3 \quad 10^{30} \\
\Delta \sigma \text { given in } \mathrm{Pa}\end{array}$} & \\
\hline
\end{tabular}

The reliability associated with the set of detail type $q$ of the structure $p$ at time $t$, after the last inspection that occurred at $T_{h}$, can be written as

$$
\begin{aligned}
R_{p q}\left(t, T_{h}\right)= & \int_{0}^{+\infty} \int_{0}^{+\infty} \int_{0}^{+\infty}\left[R_{1}\left(t, T_{h} \mid \alpha, \beta, m_{s}\right)\right]^{r_{1}} \\
& \times\left[R_{2}\left(t, T_{h} \mid \alpha, \beta, m_{s}\right)\right]^{r_{2}} f_{\alpha, \beta_{p q}, m_{s p q}} \\
& \times\left(\alpha, \beta, m_{s}\right) d \alpha d \beta d m_{s}
\end{aligned}
$$

where $r_{1}$ and $r_{2}$ are the number of details in which cracks were and were not found at the last inspection at $T_{h}$ respectively.

\section{Numerical example}

In table 1 and in table 2 are shown the numerical values and in table 3 the set of simulated inspection results used in the numerical example respectively.

There are inspections in the structures every five years, but not necessarily all detail types of a structure are inspected in a given opportunity, and the time when each structure is put into service is not fixed.

In each cell of table 3 , there are shown: when the inspection takes place ( $T=$ inspection number $\times 5$ years), the number of details found cracked, the number of details found without any cracks, and the sizes of the

Table 1 Physical parameters 
Table 2 Parameters related to uncertainty

\begin{tabular}{|l|c|c|}
\hline Coeficient of variation & Symbol & Value \\
\hline $\begin{array}{l}\text { crack propagation } \\
\text { parameter C }\end{array}$ & $\mathrm{cv}_{\mathrm{C}}$ & 0.10 \\
\hline Stress parameter S & $\mathrm{cv}_{\mathrm{S}}$ & 0.0 \\
\hline Structure effect on $\beta$ & $\mathrm{cv}_{\beta \mathrm{s}}$ & 0.20 \\
\hline Detail effect on $\beta$ & $\mathrm{cv}_{\beta \mathrm{\beta d}}$ & 0.20 \\
\hline Model Error effect on $\beta$ & $\mathrm{cv}_{\beta \mathrm{p}}$ & 0.20 \\
\hline $\begin{array}{l}\text { Structure effect on } \\
\text { the median of S }\end{array}$ & $\mathrm{cv}_{\mathrm{Ss}}$ & 0.20 \\
\hline $\begin{array}{l}\text { Detail effect on on the } \\
\text { median of S }\end{array}$ & $\mathrm{cv}_{\mathrm{Sd}}$ & 0.20 \\
\hline $\begin{array}{l}\text { Model Error effect } \\
\text { on the median of S }\end{array}$ & $\mathrm{cv}_{\mathrm{Sp}}$ & 0.15 \\
\hline
\end{tabular}

Table 3 Inspection results

\begin{tabular}{|c|c|c|c|}
\hline & Detail type 1 & Detail type 2 & Detail type 3 \\
\hline $\begin{array}{l}\overrightarrow{0} \\
\text { 氖 } \\
\text { 总 }\end{array}$ & $\begin{array}{l}\text { group } 11 \\
\text { inspection_number } 2 \\
\text { time_step } 5.0 \\
\text { time_inicio } 0.0 \\
\text { detected } 0 \\
\text { not_detected } 10\end{array}$ & $\begin{array}{l}\text { group } 12 \\
\text { inspection_number } 2 \\
\text { time_step } 5.0 \\
\text { time_inicio } 0.0 \\
\text { detected } 0 \\
\text { not_detected } 10\end{array}$ & $\begin{array}{l}\text { group } 13 \\
\text { inspection_number } 3 \\
\text { time_step } 5.0 \\
\text { time_inicio } 0.0 \\
\text { detected } 3 \\
\text { not_detected } 7 \\
\text { crack_sizes } \\
5.145076 \mathrm{e}-02 \\
2.615565 \mathrm{e}-01 \\
8.660278 \mathrm{e}-02\end{array}$ \\
\hline 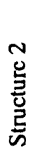 & $\begin{array}{l}\text { group } 21 \\
\text { inspection_number } 2 \\
\text { time_step } 5.0 \\
\text { time_inicio } 0.0 \\
\text { detected } 0 \\
\text { not_detected } 10\end{array}$ & $\begin{array}{l}\text { group } 22 \\
\text { inspection_number } 1 \\
\text { time_step } 5.0 \\
\text { time_inicio } 0.0 \\
\text { detected } 0 \\
\text { not_detected } 10\end{array}$ & $\begin{array}{l}\text { group } 23 \\
\text { inspection_number } 1 \\
\text { time_step } 5.0 \\
\text { time_inicio } 0.0 \\
\text { detected } 0 \\
\text { not_detected } 10\end{array}$ \\
\hline
\end{tabular}

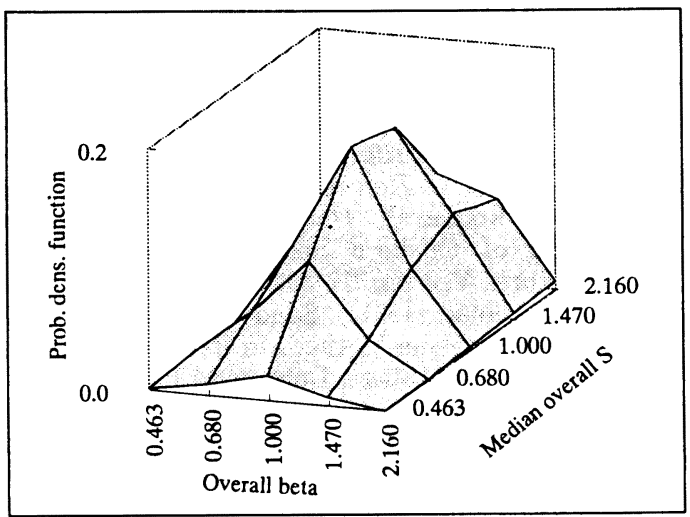

Fig. 2 Posterior distribution of $a, \bar{\beta}, \overline{m_{s}}(\alpha=4.0)$

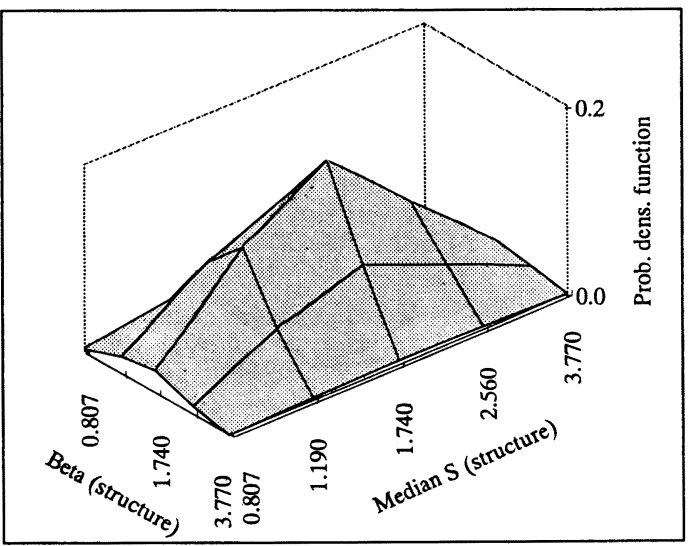

Fig. 3 Posterior distribution of $\beta_{s 1}, m_{s s 1}$ of the structure $i=1$

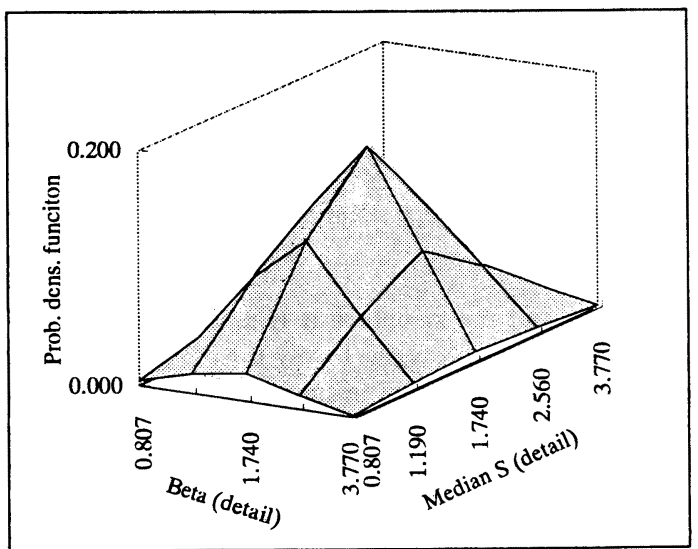

Fig. 4 Posterior distribution of $\beta_{d 3}, m_{S d 3}$ of the detail type $j=3$

cracks found (in meters).

Given the inspection results above, and using a prior distribution $f_{\alpha, \bar{\beta}, \overline{m_{s}}}^{(1)}\left(\alpha, \bar{\beta}, \overline{m_{s}}\right)$ that is uniformly distributed in the range $0.463 \leq \bar{\beta} \leq 2.160,0.463 \leq \overline{m_{s}} \leq 2.160$, $\alpha=4.0$, the joint posterior density distribution of the uncertain parameters $\alpha, \bar{\beta}, \overline{m_{s}}$ is evaluated by equation (29) and displayed in figure 2.

Now the detail type number $j=3$ of the structure number $i=1$ is considered.

Observing the data corresponding to the structure $i=$ 1 across all its details, the joint posterior probability density function for the effect of structure $i=1$ has over all its details $f_{\beta d_{1}, m_{s \alpha_{1}}}^{(1)}\left(\beta_{s_{1}}, m_{s s_{1}}\right)$ is obtained (figure 3 ).

The joint posterior degree of belief distribution of $\beta d_{3}, m_{S d_{3}}$, the effect of the detail type number 3 on fatigue life of the details of this type in both structures is show in figure 4 .

The posterior degree of belief of the modeling error term, that includes the interaction effect between the 


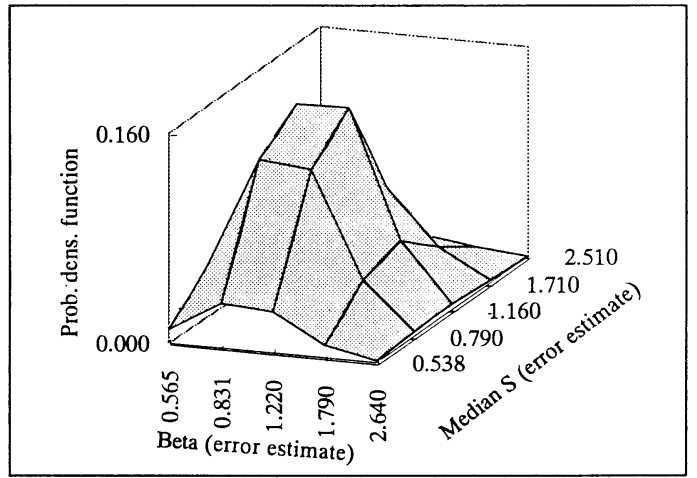

Fig. 5 Posterior distribution of $\beta_{p 1,3}, m_{S p 1,3}$

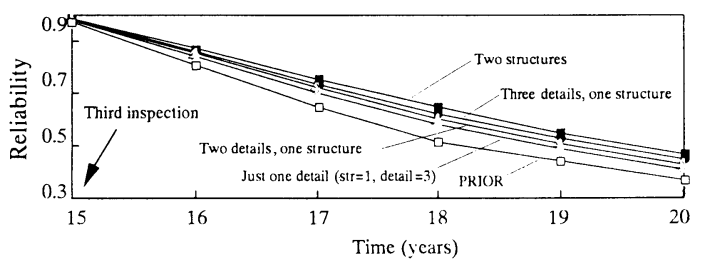

Fig. 6 Reliability

detail type and the structure it is embedded in and all other effects not accounted for by the effect of structure and detail type, is shown in figure 5 .

Now, the reliability, after the third inspection ( $T=$ 15 years after service initiation), of the detail type number 3 of the structure number 1 is evaluated considering different sets of data. The results are shown in figure 6. The condition "two structures" refers to the case analyzed above, counting all details of all structures. The case "three details, one structure" refers to the case when all data relative to the structure number 2 is ignored. In the condition "two details, one structure", only the details 2 and 3 of the structure number 1 is considered. For comparison, the reliability when the prior density distributions of all effects are used is also shown.

Note the inspection results of this particular example are highly favorable, except for the detail type 3 of the structure 1. It means that the consideration of these favorable results should in fact increase the reliability, but the point is to quantify this increase. From the results, it is clear that while there is a definite influence of the results of another structures on the one examined, it is not as strong as the consideration of details on board the same structure.

The method proposed should in principle allow one to make statements as equivalences among inspection results, regarding their effects on the structural reliability.

\section{Conclusions}

A method to infer the effects of the structure and the type of detail on the fatigue life of structural members was proposed. It is based on Bayesian methods and on some concepts of classical designs of experiments such as factorial design and decomposition of general factors into effects, including modeling error terms. A numerical test was performed and showed satisfactory results. With the information collected by using the proposed method from the inspection results, it is believed that inspection planning of fatigue sensitive structures can be carried out more effectively.

\section{References}

[Bea, 1991] Bea, R. G., Marine Structural Integrity Programs, SSC365, 1991.

[Deodatis et alli, 1992] Deodatis G., Fujimoto Y., Ito S., Spencer J., Itagaki H. : Non-Periodic Inspection by Bayesian Method I. Probabilistic Engineering Mechanics V. 7, pp. 191-204, 1992.

[Fujimoto, 1990] Fujimoto Y., Swilem A. M., Iwata M., Nagai K. : Inspection Planning for Deteorat ing Structures Based on Sequencial Cost Minimization Method. J. Soc. Naval Architects, V. 170, 1991.

[Itagaki and Yamamoto, 1985] Itagaki H., Yamamoto N.: Bayesian Analysis of Inspection on Ship Structural Members. Proceedings of ICOSSAR '85 4th International Conference on Structural Safety and Reliability, pp. (III-533) - (III-542).

[Itagaki, Ishizuka \& Yan, 1993] Itagaki H., Ishizuka T., Yan H. P. : Experimental Estimation of the Probability distribution of Fatigue Crack Growth Lives. Probabilistic Engineering Mechanics, V. 8, pp. 25-34, 1993.

[Kai-tung Ma, 1997] Kai-tung Ma, Orisamolu I. R. Bea R.G., Huang R. T., "Towards Optimal Inspection Strategies for Fatigue and Corrosion Damage", to appear.

[Kawano at alli, 1994] Selection of the First Inspection Time Based on the Maximization of Amount of Information. Kawano, A. ; Itagaki H., Ishizuka T.: Journal of The Society of Naval Architects of Japan V176, pp. 597-602, 1994.

[Lutes et alli, 1984] Lutes L. D., Corazao M., Hu S-L. J., Zimmerman J. J. : Stochastic fatigue damage accumulation. J. Struct. Engng. ASCE, 110 (ST11), pp. 2585-2601, 1984.

[Montgomery, 1976] Montgomery, D. C. "Design and Analysis of Experiments", Third ed., John Wiley \& Sons, 1976.

[Pittaluga, Cazzulo \& Romeo, 1991] Pittaluga A. Cazzulo R., Romeo P.: Uncertainties in the Fatigue Design of Offshore Steel Structures. Marine Structures V. 4 pp. 317-332, 1991.

[Sander \& Bardoux, 1991] Sander P., Bardoux R. (eds).: Bayesian Methods in Reliability. Kluwer Academic Publishers, Dordrecht, 1991.

[Sarkani et al., 1994] Sarkani S., Kihl D. P., Beach J. E. : Fatigue of Welded Joints under Narrowband non-Gaussian Loadings, Probabilistic Engineering Mechanics, V.9, pp. 179-190, 1994.

[Sobczyk K. \& Spencer, 1992] Sobczyk K., Spencer B. 
F. Jr.: Random Fatigue. Academic Press, Boston, 1992.

[Sorensen at alli, 1991] Sorensen J. D., Faber M. H. Rackwitz R., Thoft-Cristensen P.: Modelling in Optimal Inspection and Repair. Proceedings of the OMAE 1991, V. 2 Safety and Reliability.

[Vienneau, 1994] Vienneau, B. Drydock, pp. 20-25, March 1994.
[Yang and Trapp, 1974] Yang J. N. Trapp W. J. Reliability Analysis of Aircraft Structures under Random Loading and Periodic Inspection. AIAA Journal V. 12, N. 12, 1974.

[Yang et alli, 1993] Yang J. N., Manning S. D., Norton J. M. : A Stochastic Crack Growth Analysis for Aircraft Structures. Proceedings of the ICOSSAR '93, Innsbruck, Austria. 